\title{
ROTHE TIME-DISCRETIZATION METHOD FOR A NONLOCAL PROBLEM ARISING IN THERMOELASTICITY
}

NABIL MERAZGA AND ABDELFATAH BOUZIANI

Received 26 November 2003 and in revised form 21 July 2004

We investigate a model parabolic mixed problem with purely boundary integral conditions arising in the context of thermoelasticity. Using the Rothe method which is based on a semidiscretization of the given problem with respect to the time variable, the questions of existence, uniqueness, and continuous dependence upon data of a weak solution are proved. Moreover, we establish convergence and derive an error estimate for a semidiscrete approximation.

\section{Introduction}

In this paper, we make use of the Rothe time-discretization method to determine a function $v=v(x, t)$ which satisfies, in a weak sense, the diffusion equation

$$
\frac{\partial v}{\partial t}-\frac{\partial^{2} v}{\partial x^{2}}=\mathrm{f}(x, t), \quad(x, t) \in(0,1) \times(0, T]
$$

with the initial condition

$$
v(x, 0)=V_{0}(x), \quad 0 \leqslant x \leqslant 1,
$$

as well as the integral boundary conditions

$$
\begin{array}{cc}
\int_{0}^{1} v(x, t) d x=E(t), & 0 \leqslant t \leqslant T, \\
\int_{0}^{1} x v(x, t) d x=G(t), & 0 \leqslant t \leqslant T,
\end{array}
$$

where $\mathrm{f}, V_{0}, E$, and $G$ are sufficiently regular given functions of the indicated variables and $T$ is a positive constant.

This mathematical model, recently studied in [4], describes the quasistatic flexure of a thermoelastic rod, where conditions (1.3) represent, respectively, the average and weighted average of the entropy $v$. 


\section{Time-discretization method for a nonlocal problem}

Although an increasing attention has been recently given to evolution problems which involve nonlocal boundary conditions (see, for instance, $[2,3,7,8,9,10]$ and the references therein), only few works have been consecrated to mixed parabolic problems with purely integral boundary conditions over the spatial domain $[1,4,5]$. Contrary to these papers, the subject of the present paper is motivated, in essence, by our intention to develop the Rothe method to this relatively new type of evolution problems. We will point out that, in addition to the fact that Rothe's method is a convenient tool for the theoretical analysis of evolution problems, it is of particular interest from the numerical point of view.

The plan of the paper is as follows. First, in the next section, we transform problem (1.1)-(1.3) to an equivalent one with homogeneous integral conditions, namely, problem (2.3)-(2.6). Then, we specify notations and assumptions on data before stating the precise sense of the desired solution. In Section 3, by the Rothe discretization in time method, we construct approximate solutions to problem (2.3)-(2.6). Some a priori estimates for the approximations are derived in Section 4, while convergence and existence result for problem (2.3)-(2.6) are established in Section 5.

\section{Preliminaries}

It is convenient at the beginning to reduce problem (1.1)-(1.3) with inhomogeneous integral conditions (1.3) to an equivalent one with homogeneous conditions. For this, we introduce a new unknown function $u$ by setting

$$
u(x, t)=v(x, t)-R(x, t), \quad(x, t) \in(0,1) \times[0, T],
$$

where

$$
R(x, t)=6(2 G(t)-E(t)) x-2(3 G(t)-2 E(t)) .
$$

Then, the function $u$ is seen to be the solution of the following problem:

$$
\begin{gathered}
\frac{\partial u}{\partial t}-\frac{\partial^{2} u}{\partial x^{2}}=f(x, t), \quad(x, t) \in(0,1) \times(0, T], \\
u(x, 0)=U_{0}(x), \quad 0 \leqslant x \leqslant 1, \\
\int_{0}^{1} u(x, t) d x=0, \quad 0 \leqslant t \leqslant T \\
\int_{0}^{1} x u(x, t) d x=0, \quad 0 \leqslant t \leqslant T,
\end{gathered}
$$

where

$$
\begin{gathered}
f(x, t)=\mathrm{f}(x, t)-\frac{\partial R(x, t)}{\partial t}, \\
U_{0}(x)=V_{0}(x)-R(x, 0) .
\end{gathered}
$$

Hence, instead of looking for the function $v$, we search for the function $u$. The solution of problem (1.1)-(1.3) will be simply given by the formula $v(x, t)=u(x, t)+R(x, t)$. 
Throughout this paper, $(\cdot, \cdot)$ denotes the usual inner product in $L^{2}(0,1)$ and $\|\cdot\|$ the corresponding norm. $H^{2}(0,1)$ is the usual second-order Sobolev space on $(0,1)$ with norm $\|\cdot\|_{H^{2}(0,1)}$. To take into consideration the integral conditions (2.5) and (2.6), we will make use of the space $V$ which we define as follows:

$$
V:=\left\{\phi \in L^{2}(0,1) ; \int_{0}^{1} \phi(x) d x=\int_{0}^{1} x \phi(x) d x=0\right\} .
$$

Since $V$ is the null space of the continuous linear mapping $\ell: L^{2}(0,1) \rightarrow \mathbb{R}^{2}, \phi \mapsto \ell(\phi)=$ $\left(\int_{0}^{1} \phi(x) d x, \int_{0}^{1} x \phi(x) d x\right)$, it is a closed linear subspace of $L^{2}(0,1)$, consequently $V$ is a Hilbert space endowed with the inner product $(\cdot, \cdot)$.

Our analysis also requires the use of the nonclassical function space $B_{2}^{1}(0,1)$ first introduced by Bouziani in [1]. It is considered as the completion of the space $C_{0}(0,1)$ of real continuous functions with compact support in $(0,1)$ with respect to the inner product

$$
(u, v)_{B_{2}^{1}(0,1)}=\int_{0}^{1} \mathfrak{J}_{x} u \cdot \mathfrak{J}_{x} v d x
$$

where $\mathfrak{J}_{x} v=\int_{0}^{x} v(\xi) d \xi$ for every fixed $x \in(0,1)$. We recall that, if $\|\cdot\|_{B_{2}^{1}(0,1)}$ denotes the corresponding norm, that is,

$$
\|v\|_{B_{2}^{1}(0,1)}=\sqrt{(v, v)_{B_{2}^{1}(0,1)}}
$$

then, the inequality

$$
\|v\|_{B_{2}^{1}(0,1)}^{2} \leqslant \frac{1}{2}\|v\|^{2}
$$

holds for every $v \in L^{2}(0,1)$, and the embedding $L^{2}(0,1) \rightarrow B_{2}^{1}(0,1)$ is continuous.

Moreover, we need the standard functional spaces $C([0, T] ; X), C^{0,1}([0, T] ; X), L^{2}(0, T$; $X)$, and $L^{\infty}(0, T ; X)$ of continuous, Lipschitz continuous, $L^{2}$-Bochner integrable, and essentially bounded mappings from $[0, T]$ into a normed linear space $X$, respectively, see [12]. It is known that $C([0, T] ; X)$ and $L^{2}(0, T ; X)$ are complete with respect to their usual norms if $X$ is a Banach space. This is the case, for instance, when $X=V$ or $X=B_{2}^{1}(0,1)$.

Any given real function $\theta(x, t)$ on $(0,1) \times(0, T)$ is always identified with the corresponding abstract function $t \mapsto \theta(t)=\theta(\cdot, t)$ defined from $(0, T)$ into some function space on $(0,1)$ by setting $(\theta(t))(x)=\theta(x, t)$. Strong or weak convergence is denoted by $\rightarrow$ or $\rightarrow$, respectively. The letter $C$ will stand for generic positive constants which may be different in the same discussion.

The following lemma will be useful to us thereafter.

Lemma 2.1 (Lax and Milgram). Let $H$ be a real Hilbert space with norm $\|\cdot\|_{H}$. Let $(u, v) \mapsto$ $a(u, v)$ be a bilinear form on $H \times H$ and let $v \mapsto L(v)$ be a continuous linear form on $H$. Suppose that $a(\cdot, \cdot)$ is continuous and $H$-elliptic, that is, there exist two positive constants $M$ and $\alpha$ such that

(i) $|a(u, v)| \leqslant M\|u\|_{H}\|v\|_{H}$, for all $u, v \in H$;

(ii) $a(v, v) \geqslant \alpha\|v\|_{H}^{2}$, for all $v \in H$. 
Then, there exists a unique $u \in H$ satisfying

$$
a(u, v)=L(v), \quad \forall v \in H .
$$

For the proof of Lemma 2.1, see, for instance, [6, page 84].

In the sequel, we make the following assumptions:

$\left(\mathrm{H}_{1}\right) f(t) \in L^{2}(0,1)$ for each $t \in[0, T]$ and $f \in C^{0,1}\left([0, T] ; B_{2}^{1}(0,1)\right)$;

$\left(\mathrm{H}_{2}\right) U_{0} \in H^{2}(0,1)$;

$\left(\mathrm{H}_{3}\right)$ compatibility conditions $\int_{0}^{1} U_{0}(x) d x=0$ and $\int_{0}^{1} x U_{0}(x) d x=0$.

We will be concerned with a weak solution in the following sense.

Definition 2.2. Under a weak solution of problem (2.3)-(2.6), we understand a function $u:[0, T] \rightarrow L^{2}(0,1)$ such that

(i) $u \in L^{\infty}(0, T ; V) \cap C\left([0, T] ; B_{2}^{1}(0,1)\right)$;

(ii) $u$ has (a.e. in $[0, T])$ a strong derivative $d u / d t \in L^{\infty}\left(0, T ; B_{2}^{1}(0,1)\right)$;

(iii) $u(0)=U_{0}$ in $B_{2}^{1}(0,1)$;

(iv) the identity

$$
\left(\frac{d u}{d t}(t), \phi\right)_{B_{2}^{1}(0,1)}+(u(t), \phi)=(f(t), \phi)_{B_{2}^{1}(0,1)}
$$

holds for all $\phi \in V$ and a.e. $t \in[0, T]$.

Note that since $u \in C\left([0, T] ; B_{2}^{1}(0,1)\right)$, the condition (iii) makes sense, and by virtue of (i), (ii), and assumption $\left(\mathrm{H}_{1}\right)$, each term in (2.13) is well defined. On the other hand, the fulfillment of the integral conditions (2.5) and (2.6) is guaranteed by the fact that $u(t) \in V$ for a.e. $t \in[0, T]$.

\section{Construction of approximate solutions}

In order to solve problem (2.3)-(2.6) by the Rothe method, we divide the time interval $[0, T]$ into $n$ subintervals $\left[t_{j-1}, t_{j}\right], j=1, \ldots, n$, where $t_{j}=j h$ and $h=T / n$. Then, replacing the first time derivative of $u$ by the corresponding standard difference quotient, problem (2.3)-(2.6) may be approximated at each point $t=t_{j}, j=1, \ldots, n$, by the following time discretized problem.

Find a function $u_{j}:(0,1) \rightarrow \mathbb{R}$, such that

$$
\begin{gathered}
\delta u_{j}-\frac{d^{2} u_{j}}{d x^{2}}=f_{j}, \quad x \in(0,1), \\
\int_{0}^{1} u_{j}(x) d x=0, \\
\int_{0}^{1} x u_{j}(x) d x=0,
\end{gathered}
$$


where $u_{0}$ is given by

$$
u_{0}(x)=U_{0}(x), \quad x \in(0,1)
$$

and where $\delta u_{j}:=\left(u_{j}-u_{j-1}\right) / h$ and $f_{j}=f\left(\cdot, t_{j}\right)$.

Obviously, this is a recurrent system of boundary value problems, for the approximates $u_{j}$, to be solved successively for $j=1, \ldots, n$, starting from the initial function from (2.4). To prove the existence and uniqueness of such $u_{j}$, we adapt an idea of [14] in the following way: for all $j=1, \ldots, n$, we associate with problem (3.1)-(3.3) the Dirichlet boundary value problem for the second order linear ordinary differential equation

$$
\begin{gathered}
-\frac{d^{2} w_{j}}{d x^{2}}+\frac{1}{h} w_{j}=f_{j}+\frac{1}{h} w_{j-1}, \quad x \in(0,1), \\
w_{j}(0)=\lambda, \\
w_{j}(1)=\mu
\end{gathered}
$$

where $(\lambda, \mu)$ is for the moment an arbitrary, but fixed-ordered, pair of real numbers, and where $w_{0}=U_{0}$.

Since $f_{1}+(1 / h) U_{0} \in L^{2}(0,1)$, Lemma 2.1 implies the existence and uniqueness of a strong solution $w_{1} \in H^{2}(0,1)$ to the elliptic problem $(3.5)_{1}-(3.7)_{1}$. Similarly, due to the fact that $f_{2}+(1 / h) w_{1} \in L^{2}(0,1)$, by Lemma 2.1 , the existence of a unique strong solution $w_{2} \in H^{2}(0,1)$ to problem $(3.5)_{2}-(3.7)_{2}$ follows. Step by step, each $w_{j}$ is then uniquely determined in terms of $U_{0}, w_{1}, \ldots, w_{j-1}$. Thus, the following Lemma holds.

Lemma 3.1. For all $n \geqslant 1$, and for all pair $(\lambda, \mu) \in \mathbb{R}^{2}$, the auxiliary discretized problems (3.5)-(3.7), $j=1, \ldots, n$, have unique solutions $w_{j} \in H^{2}(0,1)$.

Since the functions $w_{j}$ depend on the parameters $\lambda$ and $\mu$, we will write $w_{j}(x ; \lambda, \mu)$ instead of $w_{j}(x)$. In the sequel, we wish to show that, for each $j=1, \ldots, n, \lambda$ and $\mu$ can be chosen in a suitable way such that the corresponding function $w_{j}(\cdot ; \lambda, \mu)$ is a solution of problem (3.1)-(3.3) provided that $n$ is large enough.

Evidently, the function $w_{j}(\cdot ; \lambda, \mu)$ will be a solution to problem (3.1)-(3.3) if and only if the pair $(\lambda, \mu)$ is a solution to the following system of equations:

$$
\begin{aligned}
& \int_{0}^{1} w_{j}(x ; \lambda, \mu) d x=0, \\
& \int_{0}^{1} x w_{j}(x ; \lambda, \mu) d x=0,
\end{aligned}
$$

that is, solving (3.8) will provide all the solutions to problem (3.1)-(3.3). If, in particular, (3.8) admits a unique solution, so is problem (3.1)-(3.3). The solvability of system (3.8) needs the explicit expression of $w_{j}(\cdot ; \lambda, \mu)$ in terms of $\lambda$ and $\mu$. It is clear that $w_{j}(\cdot ; \lambda, \mu)$ can be obtained as the sum of two functions $\tilde{w}_{j}$ and $\bar{w}_{j}$, where $\tilde{w}_{j}$ (dependent on neither 
18 Time-discretization method for a nonlocal problem

$\lambda$ nor $\mu$ ) and $\bar{w}_{j}$ are the solutions to the following problems, respectively:

$$
\begin{gathered}
-\frac{d^{2} \tilde{w}_{j}}{d x^{2}}+\frac{1}{h} \widetilde{w}_{j}=f_{j}+\frac{1}{h} w_{j-1}, \quad x \in(0,1), \\
\tilde{w}_{j}(0)=0 \\
\tilde{w}_{j}(1)=0 \\
-\frac{d^{2} \bar{w}_{j}}{d x^{2}}+\frac{1}{h} \bar{w}_{j}=0, \quad x \in(0,1), \\
\bar{w}_{j}(0)=\lambda, \\
\bar{w}_{j}(1)=\mu .
\end{gathered}
$$

One easily checks that $\bar{w}_{j}$ is given by

$$
\bar{w}_{j}(x)=c_{1} e^{x / \sqrt{h}}+c_{2} e^{-x / \sqrt{h}}
$$

where $c_{1}$ and $c_{2}$ are two real constants to be selected such that $\bar{w}_{j}(0)=\lambda$ and $\bar{w}_{j}(1)=\mu$ hold. An easy computation leads to

$$
c_{1}=\frac{\mu-\lambda e^{-1 / \sqrt{h}}}{e^{1 / \sqrt{h}}-e^{-1 / \sqrt{h}}}, \quad c_{2}=\frac{\lambda e^{1 / \sqrt{h}}-\mu}{e^{1 / \sqrt{h}}-e^{-1 / \sqrt{h}}} .
$$

Substituting (3.10) in (3.8), we get

$$
\begin{gathered}
\int_{0}^{1} \tilde{w}_{j}(x) d x+c_{1} \int_{0}^{1} e^{x / \sqrt{h}} d x+c_{2} \int_{0}^{1} e^{-x / \sqrt{h}} d x=0 \\
\int_{0}^{1} x \tilde{w}_{j}(x) d x+c_{1} \int_{0}^{1} x e^{x / \sqrt{h}} d x+c_{2} \int_{0}^{1} x e^{-x / \sqrt{h}} d x=0
\end{gathered}
$$

Computing the integrals and performing some elementary simplifications taking into account (3.11), we finally obtain the equivalent linear algebraic system

$$
\begin{gathered}
\lambda+\mu=\frac{\sinh (1 / \sqrt{h})}{\sqrt{h}(1-\cosh (1 / \sqrt{h}))} \int_{0}^{1} \tilde{w}_{j}(x) d x \\
\left(1-\sqrt{h} \sinh \frac{1}{\sqrt{h}}\right) \lambda+\left(\sqrt{h} \sinh \frac{1}{\sqrt{h}}-\cosh \frac{1}{\sqrt{h}}\right) \mu=\frac{\sinh (1 / \sqrt{h})}{\sqrt{h}} \int_{0}^{1} x \tilde{w}_{j}(x) d x,
\end{gathered}
$$

whose determinant

$$
\Phi(h)=2 \sqrt{h} \sinh \frac{1}{\sqrt{h}}-\cosh \frac{1}{\sqrt{h}}-1
$$

vanishes only for the value $h=\bar{h} \simeq 3.448 \times 10^{15}$. Hence, for all $h<h_{0}:=\min \{\bar{h}, T\}$, the system (3.13), and consequently (3.8), admits a unique solution $\left(\lambda_{j}, \mu_{j}\right)$ in $\mathbb{R}^{2}$. Thus, if $n_{0}$ denotes the smallest positive integer satisfying $T / n_{0} \leqslant h_{0}$, we can state the following result. 
Theorem 3.2. For all $n>n_{0}$ and for all $j=1, \ldots, n$, problem (3.1)-(3.3) admits a unique solution $u_{j}$ in $H^{2}(0,1)$, namely,

$$
u_{j}(x)=w_{j}\left(x ; \lambda_{j}, \mu_{j}\right), \quad x \in(0,1),
$$

where $w_{j}\left(x ; \lambda_{j}, \mu_{j}\right)$ is the solution of (3.5)-(3.7) with $\left(\lambda_{j}, \mu_{j}\right)$ being the unique solution of the linear system (3.13).

Now, for all $n>n_{0}$, we introduce the Rothe function $u^{(n)}:[0, T] \rightarrow H^{2}(0,1) \cap V$ defined by

$$
u^{(n)}(t)=u_{j-1}+\delta u_{j}\left(t-t_{j-1}\right), \quad t \in\left[t_{j-1}, t_{j}\right], j=1, \ldots, n,
$$

and the corresponding step function $\bar{u}^{(n)}:[0, T] \rightarrow H^{2}(0,1) \cap V$ defined as follows:

$$
\bar{u}^{(n)}(0)=U_{0}, \quad \bar{u}^{(n)}(t)=u_{j} \quad \text { for } t \in\left(t_{j-1}, t_{j}\right], j=1, \ldots, n .
$$

\section{A priori estimates for the approximations}

It seems plausible that for $n \rightarrow \infty$ (i.e., $h \rightarrow 0$ ) the limit function (in the sense given later) of the sequence $\left\{u^{(n)}\right\}_{n>n_{0}}$ will be the required solution of our problem (2.3)-(2.6). The establishment of this fact requires some a priori estimates which are based on the following lemma.

Lemma 4.1. There exists $C>0$ such that, for all $n>n_{0}$, the solutions $u_{j}$ of the time-discretized problem (3.1)-(3.3), $j=1, \ldots, n$, satisfy the estimates

$$
\begin{gathered}
\left\|u_{j}\right\| \leqslant C, \\
\left\|\delta u_{j}\right\|_{B_{2}^{1}(0,1)} \leqslant C .
\end{gathered}
$$

Proof. To derive these estimates, we need to write problem (3.1)-(3.3) in a variational formulation.

Suppose $n>n_{0}$ and let $\phi$ be any function from the space $V$ defined in (2.8). A standard integration by parts yields

$$
\int_{0}^{x}(x-\xi) \phi(\xi) d \xi=\mathfrak{J}_{x}^{2} \phi, \quad \forall x \in(0,1),
$$

where

$$
\mathfrak{J}_{x}^{2} \phi:=\mathfrak{I}_{x}\left(\mathfrak{J}_{\xi} \phi\right)=\int_{0}^{x} d \xi \int_{0}^{\xi} \phi(\eta) d \eta .
$$

Hence, taking $x=1$ in (4.3), we get

$$
\mathfrak{J}_{1}^{2} \phi=\int_{0}^{1}(1-\xi) \phi(\xi) d \xi=\int_{0}^{1} \phi(\xi) d \xi-\int_{0}^{1} \xi \phi(\xi) d \xi=0 .
$$


20 Time-discretization method for a nonlocal problem

Next, multiplying for all $j=1, \ldots, n,(3.1)$ by $\mathfrak{J}_{x}^{2} \phi$ and integrating over $(0,1)$, we get

$$
\int_{0}^{1} \delta u_{j}(x) \mathfrak{J}_{x}^{2} \phi d x-\int_{0}^{1} \frac{d^{2} u_{j}}{d x^{2}}(x) \mathfrak{J}_{x}^{2} \phi d x=\int_{0}^{1} f_{j}(x) \mathfrak{J}_{x}^{2} \phi d x .
$$

Performing some integrations by parts and using (4.5), we obtain, for each term in (4.6),

$$
\begin{aligned}
& \int_{0}^{1} \delta u_{j}(x) \mathfrak{J}_{x}^{2} \phi d x=\int_{0}^{1} \frac{d}{d x}\left(\mathfrak{J}_{x}\left(\delta u_{j}\right)\right) \mathfrak{J}_{x}^{2} \phi d x \\
& =\left.\mathfrak{J}_{x}\left(\delta u_{j}\right) \mathfrak{I}_{x}^{2} \phi\right|_{x=0} ^{x=1}-\int_{0}^{1} \mathfrak{J}_{x}\left(\delta u_{j}\right) \mathfrak{J}_{x} \phi d x \\
& =-\left(\delta u_{j}, \phi\right)_{B_{2}^{1}(0,1)} \text {, } \\
& \int_{0}^{1} \frac{d^{2} u_{j}}{d x^{2}}(x) \mathfrak{I}_{x}^{2} \phi d x=\left.\frac{d u_{j}}{d x}(x) \mathfrak{I}_{x}^{2} \phi\right|_{x=0} ^{x=1}-\int_{0}^{1} \frac{d u_{j}}{d x}(x) \mathfrak{I}_{x} \phi d x \\
& =-\int_{0}^{1} \frac{d u_{j}}{d x}(x) \mathfrak{J}_{x} \phi d x \\
& =-\left.u_{j}(x) \mathfrak{I}_{x} \phi\right|_{x=0} ^{x=1}+\int_{0}^{1} u_{j}(x) \phi(x) d x \\
& =\left(u_{j}, \phi\right) \text {, } \\
& \int_{0}^{1} f_{j}(x) \mathfrak{J}_{x}^{2} \phi d x=\int_{0}^{1} \frac{d}{d x}\left(\mathfrak{J}_{x} f_{j}\right) \mathfrak{J}_{x}^{2} \phi d x \\
& =\left.\mathfrak{J}_{x} f_{j} \mathfrak{J}_{x}^{2} \phi\right|_{x=0} ^{x=1}-\int_{0}^{1} \mathfrak{J}_{x} f_{j} \mathfrak{J}_{x} \phi d x \\
& =-\left(f_{j}, \phi\right)_{B_{2}^{1}(0,1)} \text {, }
\end{aligned}
$$

so that (4.6) becomes finally

$$
\left(\delta u_{j}, \phi\right)_{B_{2}^{1}(0,1)}+\left(u_{j}, \phi\right)=\left(f_{j}, \phi\right)_{B_{2}^{1}(0,1)}, \quad \forall j=1, \ldots, n .
$$

Particularly, from $(4.8)_{1}$, one obtains

$$
\left(\delta u_{1}, \phi\right)_{B_{2}^{1}(0,1)}+h\left(\delta u_{1}, \phi\right)=\left(f_{1}, \phi\right)_{B_{2}^{1}(0,1)}-\left(U_{0}, \phi\right) .
$$

Then, integrating by parts the second term in the right-hand side, it follows that

$$
\begin{aligned}
\left(U_{0}, \phi\right) & =\int_{0}^{1} U_{0}(x) \frac{d}{d x}\left(\mathfrak{J}_{x} \phi\right) d x \\
& =\left.U_{0}(x) \mathfrak{I}_{x} \phi\right|_{x=0} ^{x=1}-\int_{0}^{1} \frac{d U_{0}}{d x}(x) \mathfrak{I}_{x} \phi d x \\
& =-\int_{0}^{1} \frac{d U_{0}}{d x}(x) \mathfrak{I}_{x} \phi d x,
\end{aligned}
$$

but

$$
\mathfrak{J}_{x}\left(\frac{d^{2} U_{0}}{d x^{2}}\right)=\frac{d U_{0}}{d x}(x)-\frac{d U_{0}}{d x}(0), \quad \forall x \in(0,1),
$$


whence, due to (4.5),

$$
\begin{aligned}
\left(U_{0}, \phi\right) & =-\int_{0}^{1} \mathfrak{I}_{x}\left(\frac{d^{2} U_{0}}{d x^{2}}\right) \mathfrak{I}_{x} \phi d x-\frac{d U_{0}}{d x}(0) \mathfrak{I}_{1}^{2} \phi \\
& =-\int_{0}^{1} \mathfrak{J}_{x}\left(\frac{d^{2} U_{0}}{d x^{2}}\right) \mathfrak{I}_{x} \phi d x \\
& =-\left(\frac{d^{2} U_{0}}{d x^{2}}, \phi\right)_{B_{2}^{1}(0,1)},
\end{aligned}
$$

in light of which, (4.9) becomes

$$
\left(\delta u_{1}, \phi\right)_{B_{2}^{1}(0,1)}+h\left(\delta u_{1}, \phi\right)=\left(f_{1}+\frac{d^{2} U_{0}}{d x^{2}}, \phi\right)_{B_{2}^{1}(0,1)} .
$$

Testing this identity with $\phi=\delta u_{1}=\left(u_{1}-U_{0}\right) / h$ which is in $V$, thanks to $(3.2)_{1}-(3.3)_{1}$ and assumption $\left(\mathrm{H}_{3}\right)$, we get, with the help of Cauchy-Schwarz inequality,

$$
\left\|\delta u_{1}\right\|_{B_{2}^{1}(0,1)}^{2}+h\left\|\delta u_{1}\right\|^{2} \leqslant\left[\left\|f_{1}\right\|_{B_{2}^{1}(0,1)}+\left\|\frac{d^{2} U_{0}}{d x^{2}}\right\|_{B_{2}^{1}(0,1)}\right]\left\|\delta u_{1}\right\|_{B_{2}^{1}(0,1)} .
$$

Consequently,

$$
\left\|\delta u_{1}\right\|_{B_{2}^{1}(0,1)} \leqslant\|f\|_{C\left([0, T] ; B_{2}^{1}(0,1)\right)}+\left\|\frac{d^{2} U_{0}}{d x^{2}}\right\|_{B_{2}^{1}(0,1)} .
$$

On the other hand, taking the difference of relations $(4.8)_{j}-(4.8)_{j-1}, j=2, \ldots, n$, tested with $\phi=\delta u_{j}$ which belongs to $V$, in view of (3.2)-(3.3) and $(3.2)_{j-1}-(3.3)_{j-1}$, we have

$$
\left\|\delta u_{j}\right\|_{B_{2}^{1}(0,1)}^{2}+\frac{1}{h}\left\|u_{j}-u_{j-1}\right\|^{2}=\left(f_{j}-f_{j-1}, \delta u_{j}\right)_{B_{2}^{1}(0,1)}+\left(\delta u_{j-1}, \delta u_{j}\right)_{B_{2}^{1}(0,1)} .
$$

Hence, using Cauchy-Schwarz inequality,

$$
\left\|\delta u_{j}\right\|_{B_{2}^{1}(0,1)}^{2} \leqslant\left\|f_{j}-f_{j-1}\right\|_{B_{2}^{1}(0,1)}\left\|\delta u_{j}\right\|_{B_{2}^{1}(0,1)}+\left\|\delta u_{j-1}\right\|_{B_{2}^{1}(0,1)}\left\|\delta u_{j}\right\|_{B_{2}^{1}(0,1)},
$$

accordingly, due to assumption $\left(\mathrm{H}_{1}\right)$, we get

$$
\left\|\delta u_{j}\right\|_{B_{2}^{1}(0,1)} \leqslant l h+\left\|\delta u_{j-1}\right\|_{B_{2}^{1}(0,1)},
$$

where $l$ is the constant of Lipschitz continuity of $f:[0, T] \rightarrow B_{2}^{1}(0,1)$. Then, iterating this inequality, we arrive at

$$
\left\|\delta u_{j}\right\|_{B_{2}^{1}(0,1)} \leqslant l(j-1) h+\left\|\delta u_{1}\right\|_{B_{2}^{1}(0,1)}
$$

or, by virtue of (4.15),

$$
\left\|\delta u_{j}\right\|_{B_{2}^{1}(0,1)} \leqslant l T+\|f\|_{C\left([0, T] ; B_{2}^{1}(0,1)\right)}+\left\|\frac{d^{2} U_{0}}{d x^{2}}\right\|_{B_{2}^{1}(0,1)},
$$


22 Time-discretization method for a nonlocal problem

for every $j=1, \ldots, n$. Thus, (4.2) is proved with

$$
C=C_{1}:=l T+\|f\|_{C\left([0, T] ; B_{2}^{1}(0,1)\right)}+\left\|\frac{d^{2} U_{0}}{d x^{2}}\right\|_{B_{2}^{1}(0,1)} .
$$

As for estimate (4.1), we put $\phi=u_{j}-u_{j-1}$ in (4.8) and we apply the identity

$$
\left(u_{j}, u_{j}-u_{j-1}\right)=\frac{1}{2}\left(\left\|u_{j}-u_{j-1}\right\|^{2}+\left\|u_{j}\right\|^{2}-\left\|u_{j-1}\right\|^{2}\right),
$$

to get

$$
h\left\|\delta u_{j}\right\|_{B_{2}^{1}(0,1)}^{2}+\frac{1}{2}\left\|u_{j}-u_{j-1}\right\|^{2}+\frac{1}{2}\left\|u_{j}\right\|^{2}=\left(f_{j}, u_{j}-u_{j-1}\right)_{B_{2}^{1}(0,1)}+\frac{1}{2}\left\|u_{j-1}\right\|^{2} .
$$

Ignoring the first two terms in the left-hand side, we have

$$
\left\|u_{j}\right\|^{2} \leqslant 2\left\|f_{j}\right\|_{B_{2}^{1}(0,1)} \mid\left\|u_{j}-u_{j-1}\right\|_{B_{2}^{1}(0,1)}+\left\|u_{j-1}\right\|^{2}
$$

whence, using (4.20),

$$
\left\|u_{j}\right\|^{2} \leqslant 2 C_{1} h\|f\|_{C\left([0, T] ; B_{2}^{1}(0,1)\right)}+\left\|u_{j-1}\right\|^{2} .
$$

From this recurrent inequality, we successively estimate

$$
\left\|u_{j}\right\|^{2} \leqslant 2 C_{1} j h\|f\|_{C\left([0, T] ; B_{2}^{1}(0,1)\right)}+\left\|U_{0}\right\|^{2},
$$

from where estimate (i) follows with

$$
C=C_{2}:=\left\{2 C_{1} T\|f\|_{C\left([0, T] ; B_{2}^{1}(0,1)\right)}+\left\|U_{0}\right\|^{2}\right\}^{1 / 2},
$$

and so the proof is complete.

COROLlary 4.2. For all $n>n_{0}$, the functions $u^{(n)}$ and $\bar{u}^{(n)}$ satisfy the inequalities

$$
\begin{gathered}
\left\|u^{(n)}(t)\right\| \leqslant C, \quad\left\|\bar{u}^{(n)}(t)\right\| \leqslant C, \quad \forall t \in[0, T], \\
\left\|\frac{d u^{(n)}}{d t}(t)\right\|_{B_{2}^{1}(0,1)} \leqslant C, \quad \text { a.e. in }[0, T] \\
\left\|\bar{u}^{(n)}(t)-u^{(n)}(t)\right\|_{B_{2}^{1}(0,1)} \leqslant C h, \quad \forall t \in[0, T] .
\end{gathered}
$$

Proof. Inequalities (4.28) are direct consequences of (4.1) with the same constant $C=C_{2}$, whereas inequalities (4.29) and (4.30) follow immediately from (4.2), also with the same constant $C=C_{1}$, noting that

$$
\begin{gathered}
\frac{d u^{(n)}}{d t}(t)=\delta u_{j}, \quad t \in\left(t_{j-1}, t_{j}\right], 1 \leqslant j \leqslant n, \\
\bar{u}^{(n)}(t)-u^{(n)}(t)=\left(t_{j}-t\right) \delta u_{j}, \quad t \in\left(t_{j-1}, t_{j}\right], 1 \leqslant j \leqslant n,
\end{gathered}
$$


and, consequently,

$$
\begin{gathered}
\left\|\frac{d u^{(n)}}{d t}(t)\right\|_{B_{2}^{1}(0,1)} \leqslant \max _{1 \leqslant j \leqslant n}\left\|\delta u_{j}\right\|_{B_{2}^{1}(0,1)}, \quad \text { a.e. in }[0, T], \\
\left\|\bar{u}^{(n)}(t)-u^{(n)}(t)\right\|_{B_{2}^{1}(0,1)} \leqslant h \max _{1 \leqslant j \leqslant n}\left\|\delta u_{j}\right\|_{B_{2}^{1}(0,1)}, \quad \forall t \in[0, T] .
\end{gathered}
$$

\section{Convergence and existence result}

For all $n>n_{0}$, the variational equations (4.8) may be rewritten in the form

$$
\left(\frac{d u^{(n)}}{d t}(t), \phi\right)_{B_{2}^{1}(0,1)}+\left(\bar{u}^{(n)}(t), \phi\right)=\left(\bar{f}^{(n)}(t), \phi\right)_{B_{2}^{1}(0,1)}
$$

for all $\phi \in V$ and a.e. $t \in[0, T]$, where $\bar{f}^{(n)}: I \rightarrow L^{2}(0,1)$ is the step function defined by

$$
\bar{f}^{(n)}(t)= \begin{cases}f_{j} & \text { if } t \in\left(t_{j-1}, t_{j}\right], j=1, \ldots, n, \\ f_{0} & \text { if } t=0\end{cases}
$$

Before carrying out the limiting process in the approximation scheme (5.1), we prove some assertions.

Theorem 5.1. The sequence $\left\{u^{(n)}\right\}_{n}$ converges in the norm of the space $C\left([0, T] ; B_{2}^{1}(0,1)\right)$ to some function $u \in C\left([0, T] ; B_{2}^{1}(0,1)\right)$ and the error estimate

$$
\left\|u^{(n)}-u\right\|_{C\left([0, T] ; B_{2}^{1}(0,1)\right)} \leqslant C h^{1 / 2}
$$

holds for all $n>n_{0}$.

Proof. The proof is quite similar to that of [13, Theorem 4.6]. To make the proof more transparent, we outline a few major steps. The basic idea consists in showing that $\left\{u^{(n)}\right\}_{n}$ is a Cauchy sequence in the Banach space $C\left([0, T] ; B_{2}^{1}(0,1)\right)$.

First, estimating the identity

$$
\left(\bar{u}^{(n)}(t), \phi\right)=\left(\bar{f}^{(n)}(t)-\frac{d u^{(n)}}{d t}(t), \phi\right)_{B_{2}^{1}(0,1)}, \quad \forall \phi \in V \text {, for a.e. } t \in[0, T],
$$

resulting from (5.1), we readily find owing to (4.29) that

$$
\left(\bar{u}^{(n)}(t), \phi\right) \leqslant C_{3}\|\phi\|_{B_{2}^{1}(0,1)}, \quad \forall \phi \in V \text {, for a.e. } t \in[0, T]
$$

with

$$
C_{3}=l T+2\|f\|_{C\left([0, T] ; B_{2}^{1}(0,1)\right)}+\left\|\frac{d^{2} U_{0}}{d x^{2}}\right\|_{B_{2}^{1}(0,1)} .
$$

Next, let $u^{(n)}$ and $u^{(m)}$ be the Rothe approximations corresponding to the step lengths $h_{n}=T / n$ and $h_{m}=T / m$, respectively, with $m, n>n_{0}$. For all $t \in(0, T]$, there exist two 
24 Time-discretization method for a nonlocal problem

integers $k=k(n)$ and $i=i(m)$ such that $t \in\left(t_{k-1}, t_{k}\right] \cap\left(t_{i-1}, t_{i}\right]$. Hence, from the Lipschitz continuity of $f$ assumed in $\left(\mathrm{H}_{1}\right)$, it follows that

$$
\left\|\bar{f}^{(n)}(t)-\bar{f}^{(m)}(t)\right\|_{B_{2}^{1}(0,1)}=\left\|f\left(t_{k}\right)-f\left(t_{i}\right)\right\|_{B_{2}^{1}(0,1)} \leqslant l\left|t_{k}-t_{i}\right|,
$$

consequently,

$$
\left\|\bar{f}^{(n)}(t)-\bar{f}^{(m)}(t)\right\|_{B_{2}^{1}(0,1)} \leqslant l\left(h_{n}+h_{m}\right), \quad \forall t \in[0, T] .
$$

Now, if we subtract $(5.1)^{m}$ from $(5.1)$ with $\phi=u^{(n)}(t)-u^{(m)}(t)(\in V)$ noting that

$$
\left(\frac{d}{d t}\left(u^{(n)}(t)-u^{(m)}(t)\right), u^{(n)}(t)-u^{(m)}(t)\right)_{B_{2}^{1}(0,1)}=\frac{1}{2} \frac{d}{d t}\left\|u^{(n)}(t)-u^{(m)}(t)\right\|_{B_{2}^{1}(0,1)}^{2},
$$

we derive after some rearrangement,

$$
\begin{aligned}
\frac{1}{2} \frac{d}{d t} \| & u^{(n)}(t)-u^{(m)}(t)\left\|_{B_{2}^{1}(0,1)}^{2}+\right\| \bar{u}^{(n)}(t)-\bar{u}^{(m)}(t) \|^{2} \\
= & \left(\bar{u}^{(n)}(t)-\bar{u}^{(m)}(t), \bar{u}^{(n)}(t)-\bar{u}^{(m)}(t)-u^{(n)}(t)+u^{(m)}(t)\right) \\
& \quad\left(\bar{f}^{(n)}(t)-\bar{f}^{(m)}(t), u^{(n)}(t)-u^{(m)}(t)\right)_{B_{2}^{1}(0,1)}, \quad \text { a.e. in }[0, T] .
\end{aligned}
$$

Therefore, by the aid of (5.5), (5.8), (4.28), and (4.30), we estimate

$$
\begin{aligned}
& \frac{1}{2} \frac{d}{d t}\left\|u^{(n)}(t)-u^{(m)}(t)\right\|_{B_{2}^{1}(0,1)}^{2}+\left\|\bar{u}^{(n)}(t)-\bar{u}^{(m)}(t)\right\|^{2} \\
& \leqslant 2 C_{3}\left(\left\|\bar{u}^{(n)}(t)-u^{(n)}(t)\right\|_{B_{2}^{1}(0,1)}+\left\|\bar{u}^{(m)}(t)-u^{(m)}(t)\right\|_{B_{2}^{1}(0,1)}\right) \\
& \quad+\left\|\bar{f}^{(n)}(t)-\bar{f}^{(m)}(t)\right\|_{B_{2}^{1}(0,1)}\left(\left\|u^{(n)}(t)\right\|_{B_{2}^{1}(0,1)}+\left\|u^{(m)}(t)\right\|_{B_{2}^{1}(0,1)}\right) \\
& \leqslant 2 C_{3}^{2}\left(h_{n}+h_{m}\right)+2 C_{2} l\left(h_{n}+h_{m}\right),
\end{aligned}
$$

hence,

$$
\frac{1}{2} \frac{d}{d t}\left\|u^{(n)}(t)-u^{(m)}(t)\right\|_{B_{2}^{1}(0,1)}^{2} \leqslant C\left(h_{n}+h_{m}\right), \quad \forall t \in[0, T]
$$

Then, integrating this last inequality between 0 and $t$, taking into account that $u^{(n)}(0)=$ $u^{(m)}(0)=U_{0}$, we derive

$$
\left\|u^{(n)}(t)-u^{(m)}(t)\right\|_{B_{2}^{1}(0,1)}^{2} \leqslant C\left(h_{n}+h_{m}\right) T, \quad \forall t \in[0, T],
$$

whence

$$
\left\|u^{(n)}-u^{(m)}\right\|_{C\left([0, T] ; B_{2}^{1}(0,1)\right)} \leqslant C \sqrt{h_{n}+h_{m}}
$$

from which we conclude that there exists $u \in C\left([0, T] ; B_{2}^{1}(0,1)\right)$ such that $u^{(n)} \rightarrow u$ in $C\left([0, T] ; B_{2}^{1}(0,1)\right)$. Besides, letting $m \rightarrow \infty$ in $(5.14)$, we obtain the required error estimate, which finishes the proof. 
Next, on the basis of Corollary 4.2, uniform convergence property from Theorem 5.1 and the continuous embedding $V \hookrightarrow B_{2}^{1}(0,1)$, [11, Lemma 1.3.15] allows us to write directly without proof.

Theorem 5.2. The function $u$ from Theorem 5.1 possesses the following properties:

(i) $u \in L^{\infty}(0, T ; V) \cap C^{0,1}\left([0, T] ; B_{2}^{1}(0,1)\right)$;

(ii) $u$ is strongly differentiable a.e. in $[0, T]$ and $d u / d t \in L^{\infty}\left(0, T ; B_{2}^{1}(0,1)\right)$;

(iii) $u^{(n)}(t), \bar{u}^{(n)}(t) \rightarrow u(t)$ in $V$ for all $t \in[0, T]$;

(iv) $d u^{(n)} / d t \rightarrow d u / d t$ in $L^{2}\left(0, T ; B_{2}^{1}(0,1)\right)$.

Now we are ready to state our existence theorem.

Theorem 5.3. The limit function $u$ from Theorem 5.1 is the unique weak solution to problem (2.3)-(2.6) in the sense of Definition 2.2. Moreover, $u$ depends continuously upon data $f$ and $U_{0}$, namely,

$$
\|u\|_{C\left([0, T] ; B_{2}^{1}(0,1)\right)} \leqslant C\left(\|f\|_{C\left([0, T] ; B_{2}^{1}(0,1)\right)}+\left\|U_{0}\right\|_{H^{2}(0,1)}\right)
$$

where $C>0$ depends neither on $f$ nor on $U_{0}$.

Proof. Firstly, one should note that conditions (i) and (ii) from Definition 2.2 are already established in Theorem 5.2(i) and (ii). Secondly, as $u^{(n)} \rightarrow u$ in $C\left([0, T] ; B_{2}^{1}(0,1)\right)$ when $n \rightarrow \infty$ and, by definition, $u^{(n)}(0)=U_{0}$, it follows that $u(0)=U_{0}$ holds in $B_{2}^{1}(0,1)$ so the initial condition (2.4) is fulfilled. It remains to show that $u$ satisfies the integral equation (2.13). Integrating $(5.1)$ over $(0, t)(0<t \leqslant T)$ and using the fact that $u^{(n)}(0)=U_{0}$, we have

$$
\left(u^{(n)}(t)-U_{0}, \phi\right)_{B_{2}^{1}(0,1)}+\int_{0}^{t}\left(\bar{u}^{(n)}(\tau), \phi\right) d \tau=\int_{0}^{t}\left(\bar{f}^{(n)}(\tau), \phi\right)_{B_{2}^{1}(0,1)} d \tau
$$

To investigate the behavior of (5.16) as $n \rightarrow \infty$, we prove some convergence statements. Since $u^{(n)}(t) \rightarrow u(t)$ in $V$ for all $t \in[0, T]$ and since for all fixed $\phi \in V$, the functional $v \mapsto(v, \phi)_{B_{2}^{1}(0,1)}$ is an element of the dual space of $V$, we have

$$
\left(u^{(n)}(t), \phi\right)_{B_{2}^{1}(0,1)} \longrightarrow(u(t), \phi)_{B_{2}^{1}(0,1)}, \quad \forall \phi \in V, \forall t \in[0, T] .
$$

On the other hand, in view of the assumed Lipschitz continuity of $f$, we have

$$
\left\|\bar{f}^{(n)}(\tau)-f(\tau)\right\|_{B_{2}^{1}(0,1)}=\left\|f\left(t_{j}\right)-f(\tau)\right\|_{B_{2}^{1}(0,1)} \leqslant l\left|t_{j}-\tau\right|,
$$

for all $\tau \in\left(t_{j-1}, t_{j}\right], 1 \leqslant j \leqslant n$, and hence,

$$
\left\|\bar{f}^{(n)}(\tau)-f(\tau)\right\|_{B_{2}^{1}(0,1)} \leqslant \frac{C}{n} \underset{n \rightarrow \infty}{\longrightarrow} 0, \quad \forall \tau \in[0, T]
$$

therefore,

$$
\left(\bar{f}^{(n)}(\tau), \phi\right)_{B_{2}^{1}(0,1)} \longrightarrow(f(\tau), \phi)_{B_{2}^{1}(0,1)}, \quad \forall \phi \in V, \forall \tau \in[0, T]
$$


Moreover, from the definition of $\bar{f}^{(n)}$, we have, for all $\tau \in[0, T]$,

$$
\left|\left(\bar{f}^{(n)}(\tau), \phi\right)_{B_{2}^{1}(0,1)}\right| \leqslant\left\|\bar{f}^{(n)}(\tau)\right\|_{B_{2}^{1}(0,1)}\|\phi\|_{B_{2}^{1}(0,1)} \leqslant \max _{0 \leqslant j \leqslant n}\left\|f_{j}\right\|_{B_{2}^{1}(0,1)}\|\phi\|_{B_{2}^{1}(0,1)},
$$

hence,

$$
\left|\left(\bar{f}^{(n)}(\tau), \phi\right)_{B_{2}^{1}(0,1)}\right| \leqslant\|f\|_{C\left([0, T] ; B_{2}^{1}(0,1)\right)}\|\phi\|_{B_{2}^{1}(0,1)}, \quad \forall \tau \in[0, T], \forall n>n_{0} .
$$

Now, due to (5.5) and (5.22), the sequences $\left\{\left(\bar{u}^{(n)}(\tau), \phi\right)\right\}_{n}$ and $\left\{\left(\bar{f}^{(n)}(\tau), \phi\right)_{B_{2}^{1}(0,1)}\right\}_{n}$ are uniformly bounded with respect to both $\tau$ and $n$, so the Lebesgue theorem of majorized convergence is applicable to the convergence statement (iii) from Theorem 5.2 as well as to $(5.20)$, giving

$$
\begin{gathered}
\int_{0}^{t}\left(\bar{u}^{(n)}(\tau), \phi\right) d \tau \\
\int_{0}^{t}\left(\int_{0}^{t}(u(\tau), \phi) d \tau,\right. \\
(\tau), \phi)_{B_{2}^{1}(0,1)} d \tau \longrightarrow \int_{0}^{t}(f(\tau), \phi)_{B_{2}^{1}(0,1)} d \tau,
\end{gathered}
$$

as $n \rightarrow \infty$. Then, passing to the limit $n \rightarrow \infty$ in (5.16), we obtain, owing to (5.17) and $(5.23)$

$$
\left(u(t)-U_{0}, \phi\right)_{B_{2}^{1}(0,1)}+\int_{0}^{t}(u(\tau), \phi) d \tau=\int_{0}^{t}(f(\tau), \phi)_{B_{2}^{1}(0,1)} d \tau
$$

for all $\phi \in V$ and $t \in[0, T]$. Finally, differentiating (5.24) with respect to $t$ having in mind that $u:[0, T] \rightarrow B_{2}^{1}(0,1)$ is strongly differentiable for a.e. $t \in[0, T]$, we get the required relation (2.13) due to the identity

$$
\frac{d}{d t}(u(t), \phi)_{B_{2}^{1}(0,1)}=\left(\frac{d u}{d t}(t), \phi\right)_{B_{2}^{1}(0,1)}, \quad \forall \phi \in V \text {, for a.e. } t \in[0, T] .
$$

Thus, $u$ is a weak solution to problem (2.3)-(2.6). The uniqueness may be argued in the usual manner. Indeed, let $\hat{u}$ and $\tilde{u}$ be two weak solutions of (2.3)-(2.6). Taking the difference of the relations (2.13) corresponding to $\hat{u}$ and $\tilde{u}$, tested with the function $\phi=$ $\widehat{u}(t)-\tilde{u}(t)$ and using the notation $u=\hat{u}-\tilde{u}$, we find

$$
\left(\frac{d u}{d t}(t), u(t)\right)_{B_{2}^{1}(0,1)}+\|u(t)\|^{2}=0, \quad \text { for a.e. } t \in[0, T] .
$$

Hence, integrating over $(0, t)$ taking into account that $((d u / d t)(t), u(t))_{B_{2}^{1}(0,1)}=(1 / 2)(d /$ $d t)\|u(t)\|_{B_{2}^{1}(0,1)}^{2}$ and $u(0)=0$, we get

$$
\frac{1}{2}\|u(t)\|_{B_{2}^{1}(0,1)}^{2}+\int_{0}^{t}\|u(\tau)\|^{2} d \tau=0, \quad \forall t \in[0, T],
$$

from where it follows that $\|u(t)\|_{B_{2}^{1}(0,1)}^{2}=0$, for all $t \in[0, T]$, which means that $\hat{\mathcal{u}}=\tilde{u}$. 
Next, from estimate (4.28) combined with the inequality (2.11), the uniform convergence property from Theorem 5.1 enables us to derive

$$
\|u\|_{C\left([0, T] ; B_{2}^{1}(0,1)\right)} \leqslant\left\{C_{1} T\|f\|_{C\left([0, T] ; B_{2}^{1}(0,1)\right)}+\frac{\left\|U_{0}\right\|^{2}}{2}\right\}^{1 / 2} .
$$

Hence, we conclude that the linear mapping $\left(f, U_{0}\right) \mapsto u$ from $C\left([0, T] ; B_{2}^{1}(0,1)\right) \times H^{2}(0,1)$ into $C\left([0, T] ; B_{2}^{1}(0,1)\right)$ is bounded on the unit ball

$$
\left\{\left(f, U_{0}\right) ;\|f\|_{C\left([0, T] ; B_{2}^{1}(0,1)\right)}+\left\|U_{0}\right\|_{H^{2}(0,1)} \leqslant 1\right\}
$$

and therefore continuous. The inequality (5.15) follows then for $C=\sqrt{T(l T+1)+1 / 2}$ which expresses the continuous dependence of $u$ on data $f$ and $U_{0}$, and the proof is complete.

We do not end this paper without summarizing all the obtained results into the following theorem.

Theorem 5.4. Under assumptions $\left(H_{1}\right)-\left(H_{3}\right)$, problem (2.3)-(2.6) has a unique weak solution $u$ in the sense of Definition 2.2 with additional regularity, $u \in C^{0,1}\left([0, T] ; B_{2}^{1}(0,1)\right)$.

The Rothe approximations $u^{(n)}$ possess the following convergence properties:

$$
\begin{gathered}
u^{(n)}(t) \rightarrow u(t) \quad \text { in } V, \forall t \in[0, T] ; \\
u^{(n)} \longrightarrow u \quad \text { in } C\left([0, T] ; B_{2}^{1}(0,1)\right) \text { with the rate } O\left(\frac{1}{n^{1 / 2}}\right) ; \\
\frac{d u^{(n)}}{d t} \rightarrow \frac{d u}{d t} \quad \text { in } L^{2}\left(0, T ; B_{2}^{1}(0,1)\right),
\end{gathered}
$$

as $n$ tends to infinity.

Besides, $u$ depends continuously on the right-hand side of (2.3) and on the initial function.

\section{References}

[1] A. Bouziani, Mixed problem with boundary integral conditions for a certain parabolic equation, J. Appl. Math. Stochastic Anal. 9 (1996), no. 3, 323-330.

[2] - On a third order parabolic equation with a nonlocal boundary condition, J. Appl. Math. Stochastic Anal. 13 (2000), no. 2, 181-195.

[3] On a class of nonclassical hyperbolic equations with nonlocal conditions, J. Appl. Math. Stochastic Anal. 15 (2002), no. 2, 135-153.

[4] On the quasi static flexure of a thermoelastic rod, Commun. Appl. Anal. 6 (2002), no. 4, 549-568.

[5] A. Bouziani and N.-E. Benouar, Sur un problème mixte avec uniquement des conditions aux limites intégrales pour une classe d'équations paraboliques [A mixed problem with only integral boundary conditions for a class of parabolic equations], Maghreb Math. Rev. 9 (2000), no. 1-2, 55-70 (French).

[6] H. Brezis, Analyse Fonctionnelle. Théorie et Applications [Functional Analysis. Theory and Applications], Collection Mathématiques Appliquées pour la Maîtrise, Masson, Paris, 1983.

[7] J. R. Cannon, S. Perez Esteva, and J. van der Hoek, A Galerkin procedure for the diffusion equation subject to the specification of mass, SIAM J. Numer. Anal. 24 (1987), no. 3, 499-515. 


\section{Time-discretization method for a nonlocal problem}

[8] J. R. Cannon and J. van der Hoek, Diffusion subject to the specification of mass, J. Math. Anal. Appl. 115 (1986), no. 2, 517-529.

[9] M. Dehghan, Numerical techniques for a parabolic equation subject to an overspecified boundary condition, Appl. Math. Comput. 132 (2002), no. 2-3, 299-313.

[10] Parallel techniques for a boundary value problem with non-classic boundary conditions, Appl. Math. Comput. 137 (2003), no. 2-3, 399-412.

[11] J. Kačur, Method of Rothe in Evolution Equations, Teubner-Texte zur Mathematik, vol. 80, BSB B. G. Teubner Verlagsgesellschaft, Leipzig, 1985.

[12] A. Kufner, O. John, and S. Fučík, Function Spaces, Noordhoff International Publishing, Leyden, 1977.

[13] N. Merazga and A. Bouziani, Rothe method for a mixed problem with an integral condition for the two-dimensional diffusion equation, Abstr. Appl. Anal. 2003 (2003), no. 16, 899-922.

[14] M. P. Sapagovas and R. Yu. Chegis, Boundary-value problems with nonlocal conditions, Differential Equations 23 (1987), no. 7, 858-863, translated from Differ. Uravn. 23 (1987), no. 7, $1268-1274$.

Nabil Merazga: Département de Mathématiques, Centre Universitaire Larbi Ben M’hidi, Oum El Bouaghi 04000, Algeria

E-mail address: nabilmerazga@yahoo.fr

Abdelfatah Bouziani: Département de Mathématiques, Centre Universitaire Larbi Ben M’hidi, Oum El Bouaghi 04000, Algeria

E-mail address: af_bouziani@hotmail.com 


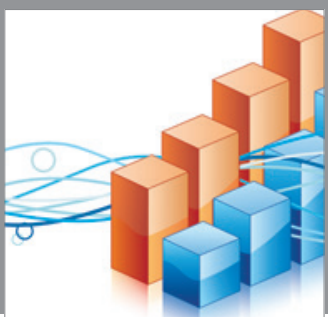

Advances in

Operations Research

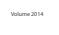

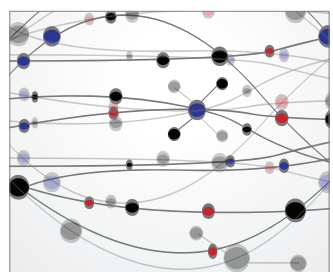

\section{The Scientific} World Journal
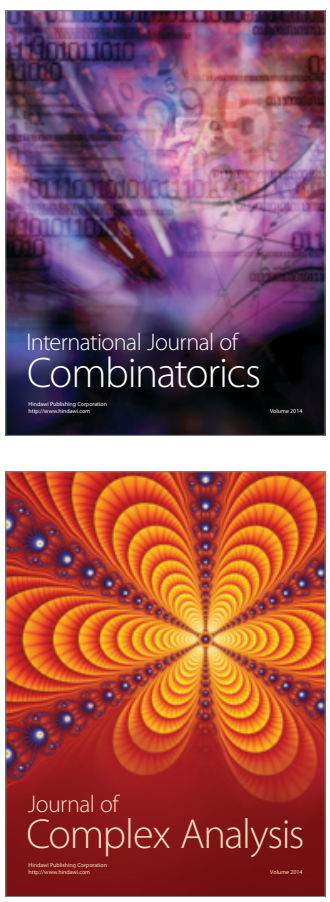

International Journal of

Mathematics and

Mathematical

Sciences
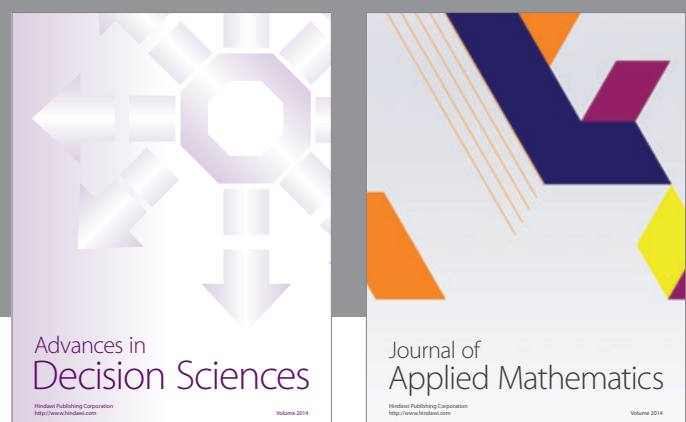

Journal of

Applied Mathematics
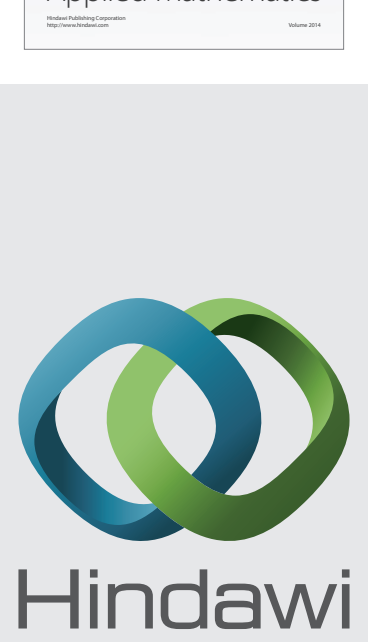

Submit your manuscripts at http://www.hindawi.com
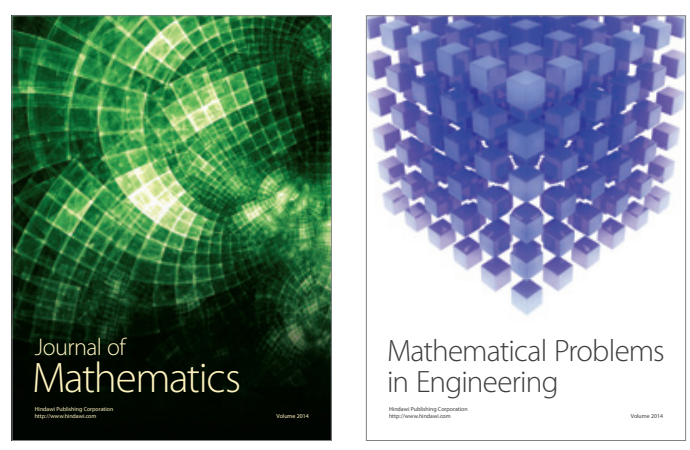

Mathematical Problems in Engineering
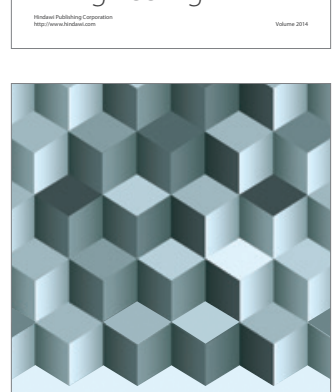

Journal of

Function Spaces
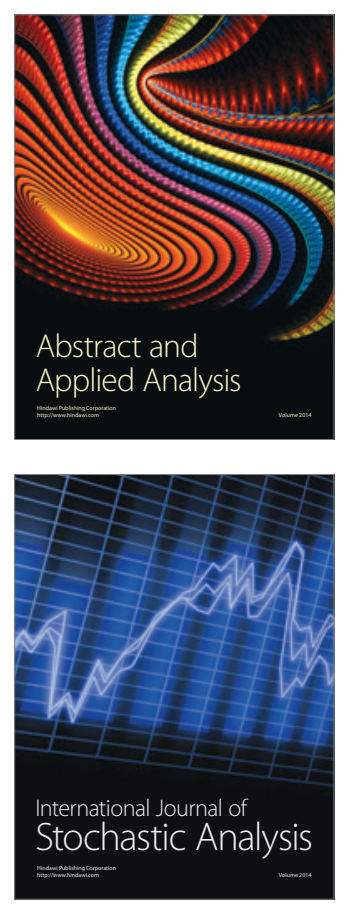

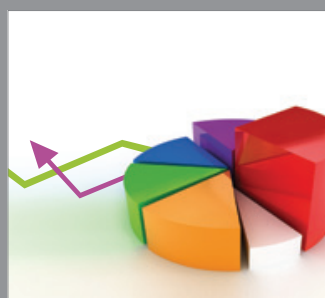

ournal of

Probability and Statistics

Promensencen
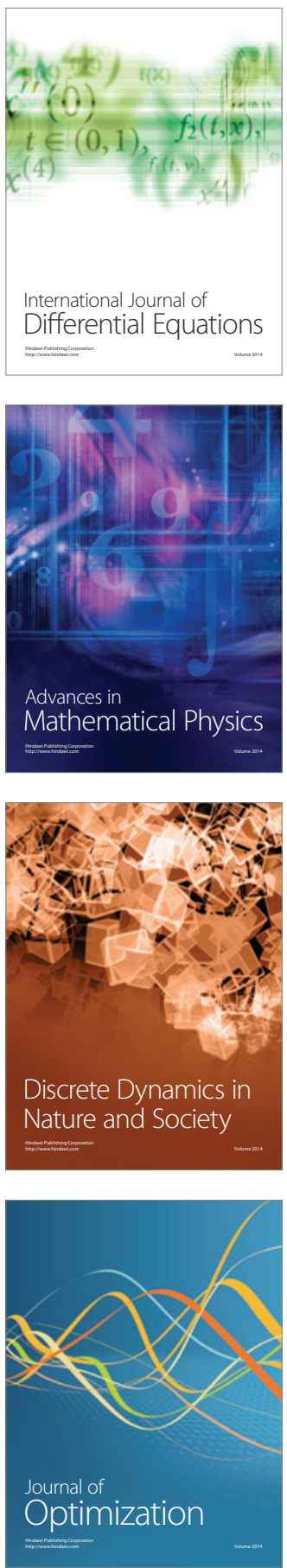